\title{
TIPE2 suppresses the tumorigenesis, growth and metastasis of breast cancer via inhibition of the AKT and p38 signaling pathways
}

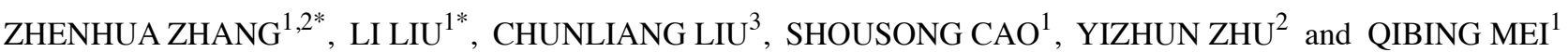 \\ ${ }^{1}$ State Institute of Pharmaceutical Industry, Shanghai 201203; ${ }^{2}$ School of Pharmacy, Fudan University, \\ Shanghai 201203; ${ }^{3}$ School of Medicine, Soochow University, Suzhou, Jiangsu 215123, P.R. China
}

Received April 29, 2016; Accepted June 21, 2016

DOI: $10.3892 / o r .2016 .5192$

\begin{abstract}
Breast cancer is the second leading cause of cancer-related deaths in female patients, and the main reasons are late diagnosis, limited therapeutic options and metastasis. Therefore, development of molecular therapeutic targets for breast cancer to suppress tumorigenesis, growth and metastasis may improve the therapeutic options and be of great benefit to patients. Tumor necrosis factor- $\alpha$-induced protein 8 -like 2 (TIPE2) is a novel molecule for maintaining immune homeostasis and is involved in cancer development. Previous studies have shown that TIPE2 plays a suppressive role in the development of several types of human cancers. However, its role in breast cancer is still not clear. In the present study, we detected TIPE2 expression in human breast cancer and adjacent normal tissues. The expression of TIPE2 was reduced in breast cancer tissues compared to the level in adjacent normal tissues. We then established a breast cancer cell line stably expressing TIPE2 to investigate the role of TIPE2 in breast cancer carcinogenesis. Our results showed that overexpression of TIPE2 significantly inhibited the proliferation of MDA-MB-231 cells as detected by Cell Counting Kit- 8 assay and suppressed the migration and invasion of breast cancer cells as detected by Transwell migration and invasion assays in vitro. TIPE2 also promoted cell apoptosis as detected by flow cytometry analysis. Moreover, TIPE2 inhibited the tumorigenesis of breast cancer in vivo. Mechanistically, TIPE2 inhibited the phosphorylation of AKT and p38 as detected by western blot analysis. Taken together, TIPE2 suppressed breast cancer tumorigenesis, growth and metastasis possibly via
\end{abstract}

Correspondence to: Dr Qibing Mei, State Institute of Pharmaceutical Industry, Shanghai 201203, P.R. China

E-mail: meiqibing2016@163.com

Dr Yizhun Zhu, School of Pharmacy, Fudan University, Shanghai 201203, P.R. China

E-mail: zhuyizhun2016@163.com

*Contributed equally

Key words: TIPE2, breast cancer, AKT, p38 regulation of the AKT and $\mathrm{p} 38$ signaling pathways. The results indicate that TIPE2 may be a potential therapeutic target for breast cancer therapy.

\section{Introduction}

Breast cancer is the most common malignancy in females worldwide (1). Breast cancerdevelops from mammary epithelial cells and interacts with the surrounding stromal tissues which are essential for malignant transformation and progression of the disease (2). It is estimated that there were 1.7 million breast cancer cases diagnosed with 521,900 mortalities worldwide in 2012 (3). Rapid progress in medical treatment has markedly improved the prognosis and early diagnosis, however, breast cancer is still the second leading cause of cancer-related deaths among female patients. The main reasons are late diagnosis, limited therapeutic options and the metastasis $(4,5)$. To date, surgery remains the most effective treatment which often has negative physiological and psychological effects on patients (6). Therefore, it is important to develop molecular therapeutic targets against the tumorigenesis, progression and metastasis of breast cancer, which have been proved to be beneficial for patients with breast cancer. However, the molecular mechanisms underlying breast cancer development and metastasis are not fully understood. Therefore, discovery of new therapeutic targets and development of novel drugs for breast cancer may increase the therapeutic options and improve the quality life and survival of these patients.

Tumor necrosis factor- $\alpha$-induced protein 8 -like-2 (TNFAIP8L2 or TIPE2) is a member of the TNFAIP8 family, and is essential for maintaining immune homeostasis $(7,8)$. Previous studies have demonstrated that TIPE2 is associated with caspase- 8 and inhibits the activation of activator protein (AP)-1 and NF- $\mathrm{KB}$. TIPE2-deficient cells showed hyper-responsiveness to Toll-like receptor and $\mathrm{T}$ cell receptor activation (9). TIPE2 was found to be involved in inflammatory and immunological diseases, such as hepatitis and systemic lupus erythematosus $(10,11)$. Downregulation of TIPE2 in humans is associated with autoimmune diseases and TIPE2 knockout in mice resulted in systemic inflammation $(8,10)$. Research has demonstrated that TIPE2 inhibits inducible nitric oxide synthase and nitric oxide production 
to suppress inflammation by switching arginine metabolism from nitric oxide synthase to arginase (12). The TIPE family, such as TNFAIP8 and TIPE1, plays important roles in most human cancers (13-15). Previous studies have also shown that TIPE2 expression is reduced or absent in various human cancers, such as gastric and lung cancer, and hepatocellular carcinoma (16-18). TIPE2 is not detected or weakly expressed in many human cancer cell lines (19). Therefore, TIPE2 may play an important role in cancer development and progression.

In the present study, we examined the expression of TIPE2 in breast cancer and their adjacent normal tissues. We also established a breast cancer cell line overexpressing TIPE2 using a lentivirus system to investigate the effects of TIPE2 on the tumorigenesis, cell growth, apoptosis, migration and invasion of breast cancer in vitro and in vivo. Furthermore, we elucidated its underlying mechanisms in breast cancer development and metastasis.

\section{Materials and methods}

Cell culture. Human embryonic kidney 293T and human breast cancer MDA-MB-231 cell lines were purchased from the Cell Bank of the Chinese Scientific Academy (Shanghai, China). 293T cells were cultured in Dulbecco's modified Eagle's medium (DMEM) with $10 \%$ fetal bovine serum (FBS). MDA-MB-231 cells were cultured in L-15 medium with $10 \%$ FBS.

Immunohistochemistry. Breast cancer tissue microarrays were purchased from Outdo Biotech (Shanghai, China). Tissue microarrays consisted of 2-mm cores with cancer and adjacent normal tissues from patients with invasive ductal carcinoma of the breast (98 cancer cases). The patient ages ranged from 36 to 74 years. Tissue microarrays were stained with rabbit anti-TIPE2 (1:100; Sigma, St. Louis, MO, USA) and HRP-conjugated anti-rabbit IgG secondary antibodies (1:1,000; MultiSciences Biotech, Hangzhou, China). DAB peroxidase substrate kit (MultiSciences Biotech) was then used to produce the enzymatic reaction. All slides were scored by evaluating the staining intensity and the percentage of positive cells as follows: staining intensity: 0 , no staining; 1 , weak staining; 2 , moderate staining; and 3 , strong staining. The percentage of positive cells was as follows: $0,<1 \% ; 1,1-33 \% ; 2,34-66 \%$; and $3,67-100 \%$. The staining intensity and the percentage of positive cells for each slide were combined to produce a final grade of TIPE2 expression score: 0 , total score $=0 ; 1+$, total score $=1-2 ; 2+$, total score $=3-4$; and $3+$, total score $=5-6$.

Plasmid and stable cell construction. The tipe2 gene was amplified from cDNA generated from human peripheral blood mononuclear cells (PBMCs). The specific primers used for the tipe 2 gene were as follows: sense, 5'-GGATCCATGGAGTCCT TCAGCTCAAAGAG-3' and antisense, 5'-GGATCCTCAGAG CTTCCCTTCGTCTAGC-3', product size, 555 bp. Then, the sequence of tipe 2 was subcloned into the lentiviral pRRL vector. The pRRL vector and lentivirus packaging plasmids were packaged in 293T cells according to the protocol (20). The TIPE2-expressing lentivirus was produced by $293 \mathrm{~T}$ cells In brief, $293 \mathrm{~T}$ cells were seeded in 10-cm-diameter dishes $24 \mathrm{~h}$ prior to transfection, and the culture DMEM was changed
2-4 h prior to transfection. For the transfection of one dish, DNA plasmids were added to $\mathrm{ddH}_{2} \mathrm{O}$ to a final volume of $450 \mu \mathrm{l} ; 50 \mu \mathrm{l}$ of $2.5 \mathrm{M} \mathrm{CaCl}_{2}$ was added to the plasmid mixture and gently mixed well, and then dropwise $500 \mu \mathrm{l}$ of $2 \mathrm{X}$ HEPES-buffered saline was added, and finally the precipitate was added to the cultures. The medium was replaced after $12-24 \mathrm{~h}$, the conditioned medium containing lentivirus was collected after $48 \mathrm{~h}$ and filtered through $0.22-\mu \mathrm{m}$ pore-size acetate filters. Then, the MDA-MB-231 cells were infected with the TIPE2-expressing-lentivirus. Empty lentiviral vector was used as a control. The transfected cells expressed GFP protein as the lentivirus included the GFP encoding sequence. In addition, the MDA-MB-231 cell line stably expressing TIPE2 or the vector control was established by sorting GFP-positive cells using a BD FACS flow cytometer (BD Biosciences, San Jose, CA, USA) and confirmed by fluorescence microscopy.

Intracellular staining. The rabbit anti-TIPE2 (1:100) and the PE goat anti-rabbit IgG secondary antibodies (1:100; MultiSciences Biotech) were used for detecting TIPE2 expression in the transfected MDA-MB-231 cells. The cells were collected and fixed with $4 \%$ formaldehyde solution, and then stained with the anti-TIPE2 antibody. Flow cytometric analyses were performed using a BD FACSCalibur flow cytometer (BD Biosciences) and analyzed using FlowJo software (Tree Star, Ashland, OR, USA).

Cell proliferation and cell apoptosis assays. Cell proliferation was determined using Cell Counting Kit-8 (CCK-8; Dojindo, Kumamoto, Japan) according to the manufacturer's instructions. MDA-MB-231/vector and MDA-MB-231/TIPE2 cells (3,000/well) were plated in a 96-well plate and subjected to CCK- 8 assay after $48 \mathrm{~h}$. CCK- 8 solution was added to each well and incubated for $4 \mathrm{~h}$. The optical density (OD) value was detected at a wavelength of $450 \mathrm{~nm}$ on a microplate reader. For cell apoptosis assay, MDA-MB-231/vector and MDA-MB-231/TIPE2 cells (3×10\%/well) were seeded into a 6 -well plate and incubated for $48 \mathrm{~h}$. Then, the cells were collected and stained with Annexin V and 7-AAD (BD Biosciences), and were analyzed by flow cytometry. The data were analyzed by FlowJo software.

Migration and invasion assays. The migration and invasion abilities of the MDA-MB-231/vector and MDA-MB-231/TIPE2 cells were assessed by Transwell assay and Transwell smeared with Matrigel assay (BD Biosciences), respectively. For both assays, MDA-MB-231/vector and MDA-MB-231/TIPE2 cells were cultured in serum-free medium in the upper chamber, wherein $1 \times 10^{5}$ cells were planted to migrate or invade through a filter $(8 \mu \mathrm{m})$ toward $10 \%$ serum-containing medium in the bottom chamber of a 24 -well plate for $16 \mathrm{~h}$ in the migration assay and for $24 \mathrm{~h}$ in the invasion assay, respectively. The migrating and invading cells that passed through the membrane were stained with $0.1 \%$ crystal violet and counted under a microscope.

Western blot analysis. Cells were lysed in the presence of protease and phosphatase inhibitors (Thremo Scientific Pierce, Rockford, IL, USA) and the total protein concentration 
was determined. Protein was separated by SDS-PAGE and transferred to polyvinylidene difluoride (PVDF) membranes (Millipore, Billerica, MA, USA). The membranes were blocked with 5\% milk in Tris-buffered saline and Tween-20 (TBST), and then incubated with primary antibodies overnight. The following primary antibodies (Cell Signaling Technology, Inc., Danvers, MA, USA) were used: anti-AKT $(1: 1,000)$ and phospho-AKT $(1: 1,000)$, anti-p38 $(1: 1,000)$ and phospho-p38 $(1: 1,000)$ and anti-GAPDH $(1: 3,000)$, followed by secondary antibodies (1:3,000; goat anti-rabbit IgG or goat anti-mouse IgG; Cell Signaling Technology, Inc.) conjugated with horseradish peroxidase for $1 \mathrm{~h}$ at room temperature. After washing, immunoreactive bands were visualized with enhanced chemiluminescence (ECL) using an ECL commercial kit (Thermo Scientific Pierce).

Experimental animals. Specific pathogen-free female BALB/c nude mice (6-8-weeks old) were purchased from the Shanghai Laboratory Animal Center (Shanghai, China). All mice were housed in specific pathogen-free facilities and in accordance with the National Animal Care and Use Committee. All animal studies were approved by the Institutional Laboratory Animal Care and Use Committee at Fudan University.

Tumor xenograft model. MDA-MB-231/vector and MDA-MB-231/TIPE2 cells ( $1 \times 10^{6}$ cells in $100 \mu \mathrm{l}$ PBS) were subcutaneously injected into the right hind legs of female nude mice. After two weeks of injection, the tumor sizes were measured every three or four days using a caliper, and the tumor volume $(\mathrm{V})$ was calculated according to the standard formula: $\mathrm{V}=1 / 2 \times$ length $\mathrm{x}$ width ${ }^{2}$. When the tumor volume reached $\sim 1,000 \mathrm{~mm}^{3}$ (35 days post injection of tumor cells), the mice were sacrificed and the tumors were removed and photographed.

Statistical method. Statistical analysis was performed using GraphPad Prism 5 software (GraphPad, San Diego, CA, USA). The data are presented as mean \pm standard deviation (SD). The data of the groups were compared with Student's t-test (unpaired, two-tailed). For all analysis, a p-value $<0.05$ was considered to indicate a statistically significant difference.

\section{Results}

Expression of TIPE2 is reduced in human breast cancer. Previous studies have shown that TIPE2 is significantly correlated with various types of human cancers, such as gastric and lung cancer, and hepatocellular carcinoma (16-18). However, the expression of TIPE 2 in breast cancer is still unclear. To determine the expression of TIPE 2 in breast cancer, we examined the levels of TIPE2 expression in breast cancer and adjacent normal tissues by immunohistochemistry. As shown in Fig. 1, the intensity and proportion of TIPE2-positive stained cells were markedly downregulated in the breast cancer tissues compared to the high levels of TIPE2 expression in the samples of adjacent normal tissues (Fig. 1A and B). The data indicate that TIPE2 may play an important role in breast carcinogenesis.

TIPE2 attenuates cell proliferation and promotes cell apoptosis. To investigate the function of TIPE2 in breast cancer,
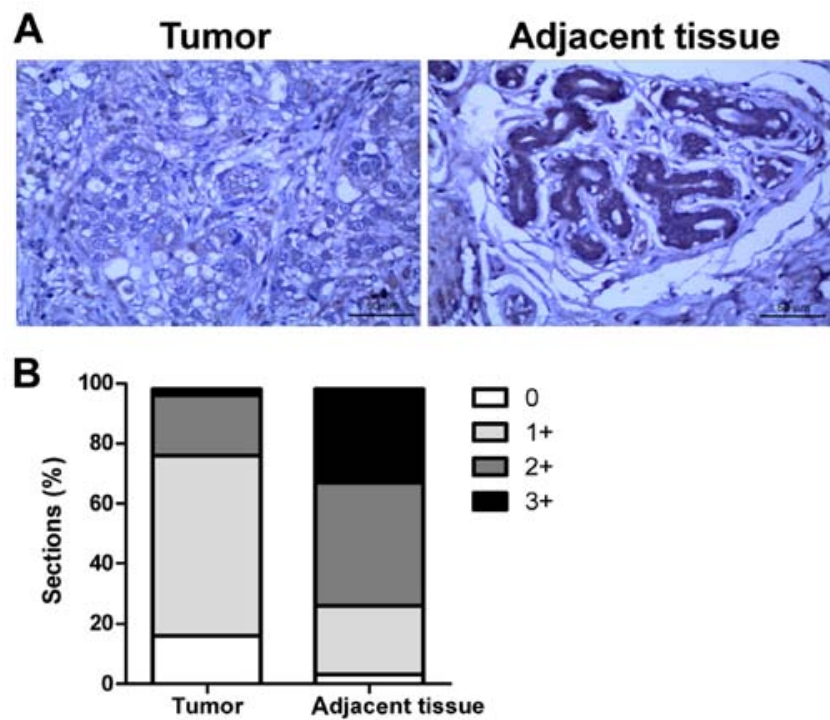

Figure 1. TIPE2 expression in human breast cancer. (A) TIPE2 expression was detected by immunohistochemistry in breast cancer and adjacent normal tissues with rabbit anti-TIPE2 antibody. TIPE2-positive cells are shown in brown. Original magnification, $x 400$. (B) All slides were scored according to the percentage and intensity of positive cells as described in Materials and methods.

A

A Visible
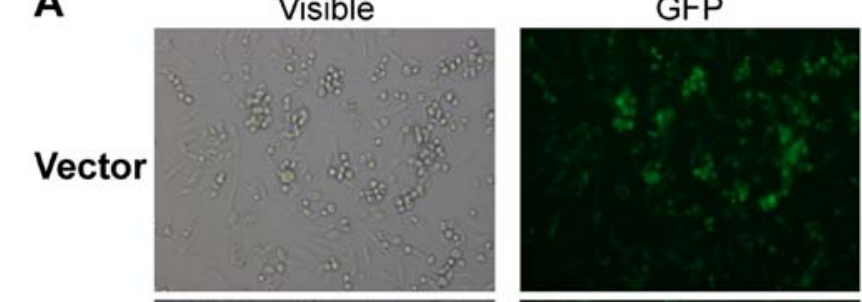

TIPE2
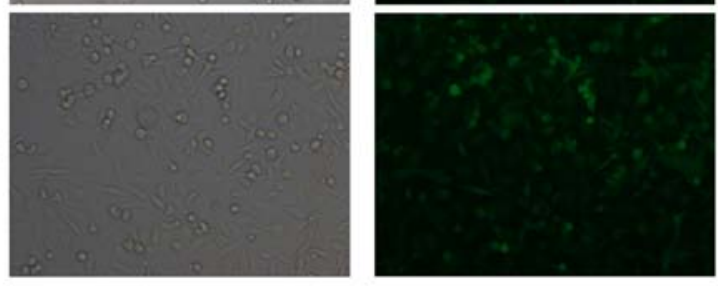

B

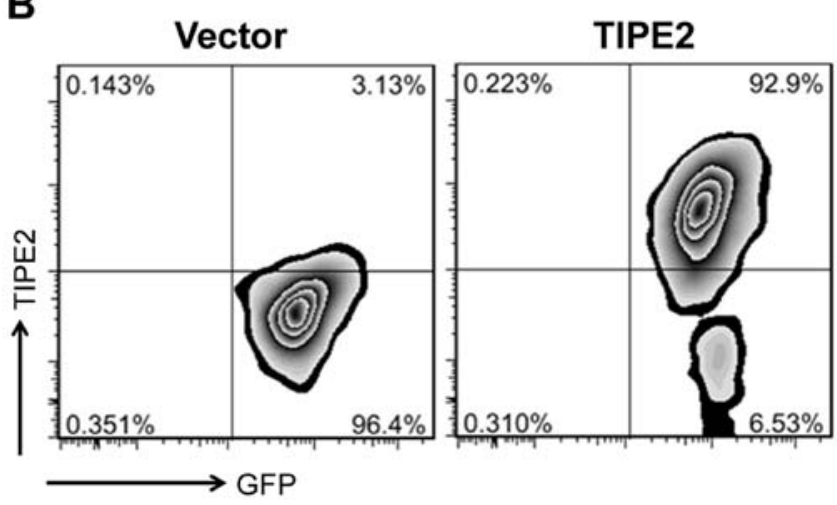

Figure 2. Overexpression of TIPE2 in breast cancer cells by lentivirus transfection. (A) A stable transfected breast cancer cell line was established by lentiviral transfection. Representative images of stable transfected cells in bright field and dark field are shown. (B) TIPE2 expression in MDA-MB-231/ vector and MDA-MB-231/TIPE2 cells by flow cytometry. Stable transfected cells were stained with rabbit anti-TIPE2 antibody and PE secondary goat anti-rabbit IgG antibody via intracellular staining. 

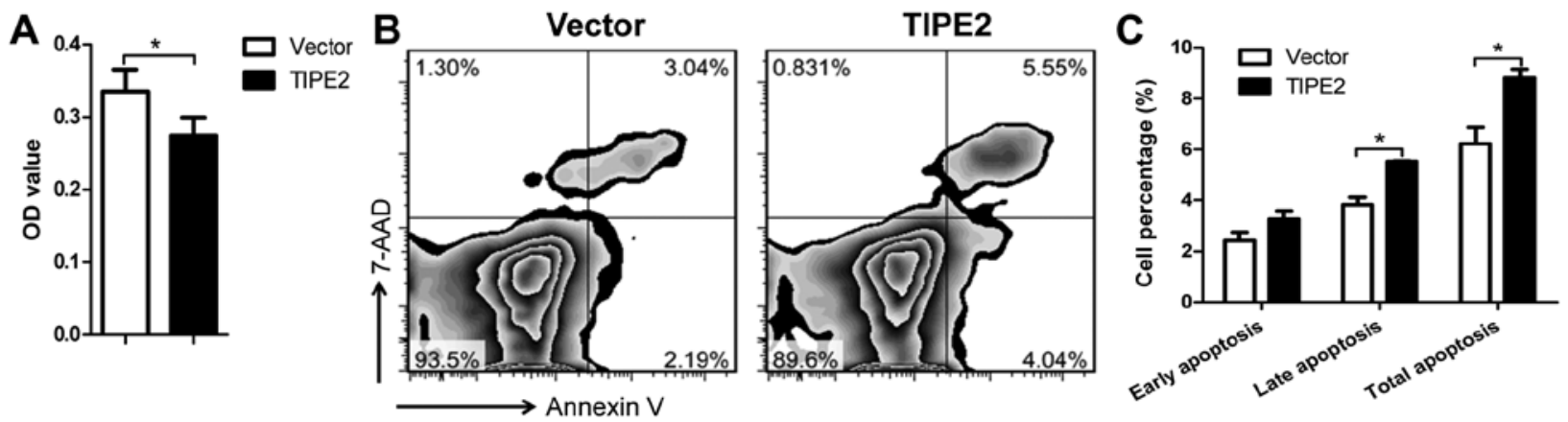

Figure 3. TIPE2 inhibits cell proliferation and promotes cell apoptosis of MDA-MB-231 cells. (A) Cell viability of MDA-MB-231 cells with stable expression of TIPE2 or vector control was determined by CCK-8 assay. (B) MDA-MB-231/vector and MDA-MB-231/TIPE2 cells were stained with Annexin V and 7-AAD to detect cell apoptosis by flow cytometry. Early apoptosis, Annexin $\mathrm{V}^{+} / 7-\mathrm{AAD}$; late apoptosis, Annexin $\mathrm{V}^{+} / 7-\mathrm{AAD}^{+}$. (C) Percentage of apoptotic MDA-MB-231/vector and MDA-MB-231/TIPE2 cells. The data shown are representative of three independent experiments; ${ }^{*} \mathrm{p}<0.05$.

A
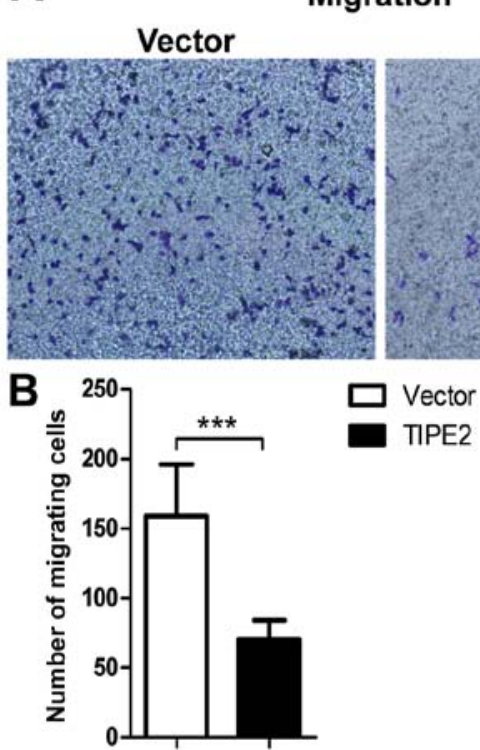

C TIPE2
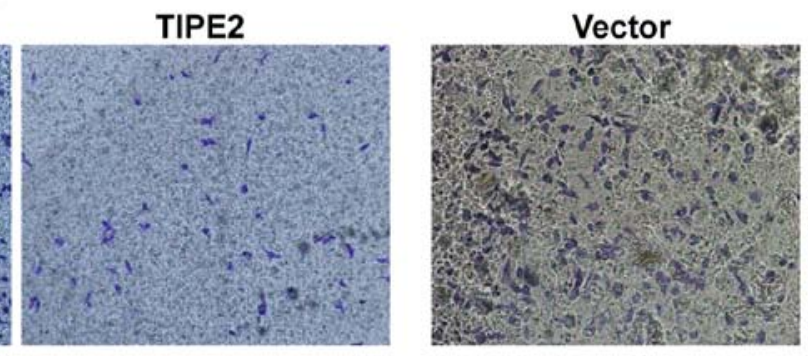

Invasion

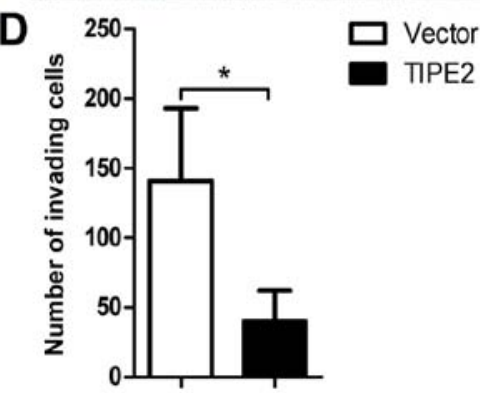

Figure 4. TIPE2 suppresses the migration and invasion of MDA-MB-231 cells. (A) MDA-MB-231/vector and MDA-MB-231/TIPE2 cells were added to the upper chamber of a 24-well Transwell. After a 16-h incubation, the tumor cells on the bottom side of the chamber were fixed and stained with crystal violet. The representative images of migrating cells are shown. (B) The cell numbers of migrating MDA-MB-231/vector and MDA-MB-231 cells. (C) MDA-MB-231/ vector and MDA-MB-231/TIPE2 cells were added to the upper chamber of Matrigel-coated 24-well Transwell. After a 24-h incubation, the tumor cells on the bottom side of the chamber were fixed and stained with crystal violet. The representative images of invading cells are shown. (D) The cell numbers of invading MDA-MB-231 cells. The data shown are representative of three independent experiments. Values are presented as means $\pm \mathrm{SD} ;{ }^{*} \mathrm{p}<0.05,{ }^{* * *} \mathrm{p}<0.001$.

we transfected breast cancer cell line MDA-MB-231 with a lentivirus expressing TIPE2 to establish cells stably expressing TIPE2 or a vector control by sorting GFP-positive cells. The expression of GFP in the transfected cells was determined by fluorescence microscopy (Fig. 2A). We found that TIPE2 was overexpressed in the transfected MDA-MB-231/TIPE2 cells compared to the vector control via intracellular staining by flow cytometry (Fig. 2B). To assess the effects of TIPE2 expression on tumor cell proliferation and apoptosis in vitro, we examined cell growth inhibition and apoptosis of the MDA-MB-231/vector and MDA-MB-231/TIPE2 cells. The cell proliferation was significantly decreased by TIPE2 expression as determined by CCK- 8 assay (Fig. 3A). The rate of apoptosis in the cells expressing TIPE2 (5.23\%) was significantly increased compared to that of the vector control cells $(9.59 \%)$ (Fig. 3B and C). These results suggest that TIPE2 markedly inhibited the cell proliferation and promoted apoptosis in the cancer cells in vitro.

TIPE2 suppresses the migration and invasion of breast cancer cells. To explore the effect of TIPE2 on breast cancer cell metastasis in vitro, we further investigated the role of TIPE2 in breast cancer cell migration and invasion by Transwell assays. Overexpression of TIPE2 significantly reduced the migrating cell number of the MDA-MB-231 cells compared to that of the vector control (Fig. 4A and B). TIPE2 also attenuated the number of cells invading through Matrigel (Fig. 4C and D). Therefore, the results indicated that TIPE2 effectively suppressed the ability of migration and invasion of the breast cancer cells. 


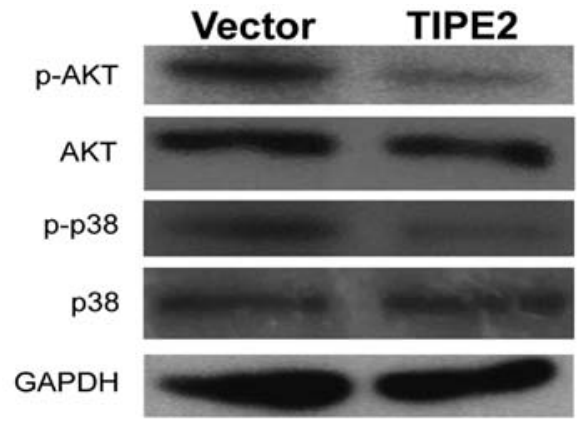

Figure 5. TIPE2 suppresses the progression of breast cancer via inhibition of the AKT and $\mathrm{p} 38$ signaling pathways. AKT and $\mathrm{p} 38$ expression was assessed using the total cellular proteins extracted from the MDA-MB-231/vector and MDA-MB-231/TIPE2 cells by western blot analysis. Then, the cellular proteins were immunoblotted with antibodies specific for AKT, p-AKT, p38, p-p38 and GAPDH.

Overexpression of TIPE2 inhibits PI3K/AKT and p38/MAPK signaling pathways. The PI3K/AKT and p38/MAPK signaling pathways are crucial to the control of cell proliferation, apoptosis and metastasis. To identify the signal transduction pathways involved in the effects of the overexpression of TIPE2 on the breast cancer cells, we examined the expression levels and activation status of AKT and p38. The expression of AKT, p-AKT, p38 and p-p38 in the MDA-MB-231/vector and MDA-MB-231/TIPE2 cells was detected by western blot analysis. TIPE2 overexpression in the MDA-MB-231 cells significantly downregulated the phosphorylation of AKT and p38 (Fig. 5). The data suggest that suppression of the development of the breast cancer cells by TIPE2 was possibly via inhibition of the AKT and p38 signaling pathways.

TIPE2 inhibits the tumorigenesis of breast cancer in vivo. To investigate the effect of TIPE2 on the tumorigenesis of breast cancer in vivo, we established subcutaneous MDA-MB-231/vector and MDA-MB-231/TIPE2 tumor xenografts in a nude mouse model by cell injection. The cells began to form measurable tumors on day 14 after inoculation. The tumor volume of the TIPE2 group was significantly less than that of the vector control group during the progression of the breast cancer (Fig. 6A). Mice were sacrificed $\sim 35$ days after tumor cell inoculation due to the large tumor volume in the control group mice. The tumors were removed from the mice and photographed. The tumor size in the vector control group was significantly larger than that in the TIPE2 group (Fig. 6B). The results demonstrated that TIPE2 significantly suppressed the tumorigenesis and progression of breast cancer in vivo.

\section{Discussion}

Previous studies have shown that the expression of TIPE2 is involved in the carcinogenesis of human cancers (16-18). TIPE2 was found to inhibit the proliferation of gastric cancer cells by upregulating the expression of $\mathrm{p} 27$, and suppressed the metastasis of gastric cancer via negatively regulating $\beta$-catenin signaling by inhibiting AKT and activating $\operatorname{GSK} 3 \beta(18,21)$. TIPE2 downregulation was found to be associated with the poor prognosis of non-small cell lung cancer, and overexpression of
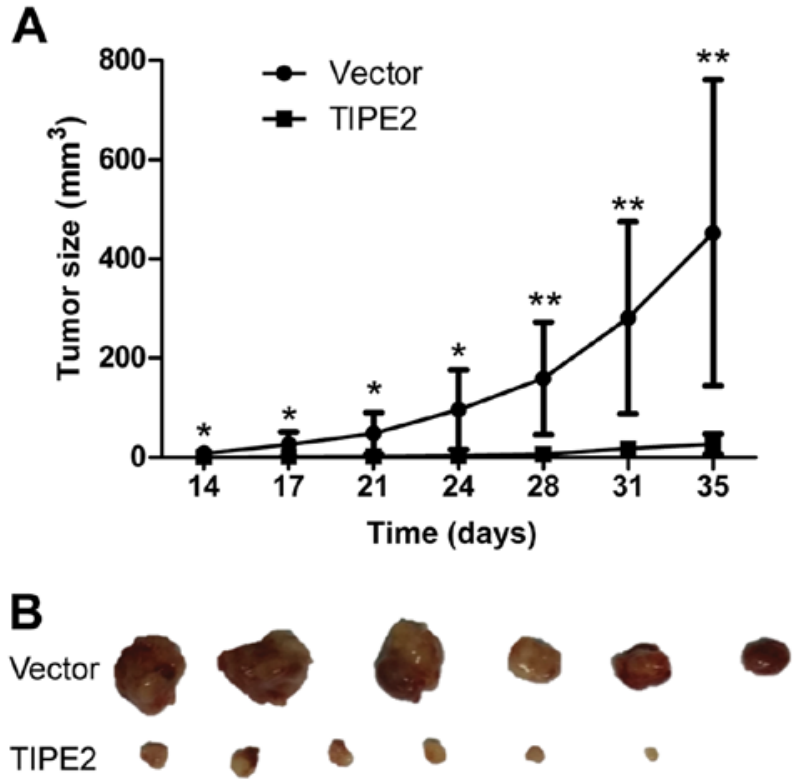

Figure 6. Overexpression of TIPE2 inhibits the tumorigenesis of breast cancer in vivo. (A) The growth curve of subcutaneous tumors in nude mice following injection with MDA-MB-231/vector and MDA-MB-231/TIPE2 cells. (B) Representative images of the tumors removed from the vector control and TIPE2 groups 35 days after tumor cell inoculation; ${ }^{*} \mathrm{p}<0.05,{ }^{* *} \mathrm{p}<0.01$.

TIPE2 inhibited cell proliferation and invasion by suppressing Bcl-xL and N-cadherin expression (17). TIPE2 was reported as an inhibitor of the oncogenic Ras and could promote cell death via competitively binding to Ras-interacting domain of RGL, thereby inhibiting RGL-induced activation of Ral GTPase and AKT. Overexpression of TIPE2 in Ras 3T3 cells significantly delayed tumor onset in vivo (16). In hepatocellular carcinoma, overexpression of TIPE2 led to hepatocellular carcinoma cell death and inhibited Ras-induced tumorigenesis (16). TIPE2 also inhibited the metastasis of hepatocellular carcinoma via suppressing Ral and Racl GTPases and reducing F-actin polymerization and expression of matrix metallopeptidase 9 (MMP9) and urokinase plasminogen activator (uPA) (22). These studies indicate that TIPE2 may exert tumor-suppressive effects against cancer development.

However, the expression of TIPE2 and its regulatory mechanisms in breast cancer are still unclear. In the present study, TIPE2 expression was found to be downregulated in breast cancer tissues compared to that in their adjacent normal tissues. Thus, we speculated that TIPE2 may affect the development of breast cancer. Then, we established a TIPE2overexpressing breast cancer cell line by lentivirus system. We found that TIPE2 overexpression inhibited the proliferation and promoted the apoptosis of the cancer cells. Furthermore, TIPE2 also suppressed the migration and invasion of the breast cancer cells in vitro. Additionally, overexpression of TIPE2 attenuated the tumorigenesis and growth of breast cancer in vivo. These results indicate that TIPE2 plays an important role in the regulation of the tumorigenesis, growth and metastasis of breast cancer.

To investigate the effect of TIPE2 on the signaling pathways in breast cancer cells, we detected the expression of several common signaling molecules in the cells overex- 
pressing TIPE2. We found that the level of phosphorylated AKT was downregulated by TIPE2. The PI3K/AKT signaling pathway plays an important role in tumorigenesis and AKT activation regulates critical cancer cell physiology, including cell survival, proliferation, metastasis, apoptosis and metabolism (23). The PI3K/AKT signaling pathway is involved in breast cancer pathogenesis, and it may be a factor for drug resistance to systemic treatments of breast cancer (24). We also found that TIPE2 decreased the expression of phosphorylated $\mathrm{p} 38$. It has been reported that $\mathrm{p} 38$ contributes to the proliferation and metastasis of breast cancer, and inhibition of p38 activation can suppress the growth and metastasis of breast cancer cells (25-27). Therefore, TIPE2 may suppress the tumorigenesis, growth and metastasis of breast cancer by inhibiting AKT and p38 phosphorylation.

Taken together, we demonstrated that TIPE2 was weakly expressed in human breast cancer compared to that observed in adjacent normal tissues. Overexpression of TIPE2 significantly suppressed the growth and metastasis of breast cancer possibly via inhibition of the activation of ATK and p38. Therefore, TIPE2 may become a potential therapeutic target for breast cancer therapy.

\section{Acknowledgements}

The present study was supported by project funding from Shanghai City (15DZ2291700).

\section{References}

1. DeSantis C, Ma J, Bryan L and Jemal A: Breast cancer statistics, 2013. CA Cancer J Clin 64: 52-62, 2014.

2. Bissell MJ and Hines WC: Why don't we get more cancer? A proposed role of the microenvironment in restraining cancer progression. Nat Med 17: 320-329, 2011.

3. Torre LA, Bray F, Siegel RL, Ferlay J, Lortet-Tieulent J and Jemal A: Global cancer statistics, 2012. CA Cancer J Clin 65: 87-108, 2015.

4. Farazi TA, Hoell JI, Morozov P and Tuschl T: MicroRNAs in human cancer. Adv Exp Med Biol 774: 1-20, 2013.

5. Filipova A, Seifrtova M, Mokry J, Dvorak J, Rezacova M, Filip S and Diaz-Garcia D: Breast cancer and cancer stem cells: A mini-review. Tumori 100: 363-369, 2014.

6. Edge SB: Quality measurement in breast cancer. J Surg Oncol 110: 509-517, 2014.

7. Lou Y and Liu S: The TIPE (TNFAIP8) family in inflammation, immunity, and cancer. Mol Immunol 49: 4-7, 2011.

8. Sun H, Gong S, Carmody RJ, Hilliard A, Li L, Sun J, Kong L, $\mathrm{Xu}$ L, Hilliard B, Hu S, et al: TIPE2, a negative regulator of innate and adaptive immunity that maintains immune homeostasis. Cell 133: 415-426, 2008.

9. Freundt EC, Bidere $\mathrm{N}$ and Lenardo MJ: A different TIPE of immune homeostasis. Cell 133: 401-402, 2008.

10. Li D, Song L, Fan Y, Li X, Li Y, Chen J, Zhu F, Guo C, Shi Y and Zhang L: Down-regulation of TIPE2 mRNA expression in peripheral blood mononuclear cells from patients with systemic lupus erythematosus. Clin Immunol 133: 422-427, 2009.
11. Wang LY, Fan YC, Zhao J, Gao S, Sun FK, Han J, Yang Y and Wang K: Elevated expression of tumour necrosis factor- $\alpha$ induced protein 8 (TNFAIP8)-like 2 mRNA in peripheral blood mononuclear cells is associated with disease progression of acute-on-chronic hepatitis B liver failure. J Viral Hepat 21: 64-73, 2014.

12. Lou Y, Zhang G, Geng M, Zhang W, Cui J and Liu S: TIPE2 negatively regulates inflammation by switching arginine metabolism from nitric oxide synthase to arginase. PLoS One 9: e96508, 2014.

13. Cui J, Zhang G, Hao C, Wang Y, Lou Y, Zhang W, Wang J and Liu S: The expression of TIPE1 in murine tissues and human cell lines. Mol Immunol 48: 1548-1555, 2011.

14. Dong QZ, Zhao Y, Liu Y, Wang Y, Zhang PX, Jiang GY, Dong XJ, Cui QZ and Wang EH: Overexpression of SCC-S2 correlates with lymph node metastasis and poor prognosis in patients with non-small-cell lung cancer. Cancer Sci 101: 1562-1569, 2010.

15. Kumar D, Gokhale P, Broustas C, Chakravarty D, Ahmad I and Kasid U: Expression of SCC-S2, an antiapoptotic molecule, correlates with enhanced proliferation and tumorigenicity of MDA-MB 435 cells. Oncogene 23: 612-616, 2004.

16. Gus-Brautbar Y, Johnson D, Zhang L, Sun H, Wang P, Zhang S, Zhang L and Chen YH: The anti-inflammatory TIPE2 is an inhibitor of the oncogenic Ras. Mol Cell 45: 610-618, 2012.

17. Li Y, Li X, Liu G, Sun R, Wang L, Wang J and Wang H: Downregulated TIPE2 is associated with poor prognosis and promotes cell proliferation in non-small cell lung cancer. Biochem Biophys Res Commun 457: 43-49, 2015.

18. Zhao Q, Zhao M, Dong T, Zhou C, Peng Y, Zhou X, Fan B, Ma W, Han M and Liu S: Tumor necrosis factor- $\alpha$-induced protein- 8 like-2 (TIPE2) upregulates p27 to decrease gastic cancer cell proliferation. J Cell Biochem 116: 1121-1129, 2015.

19. Zhang G, Hao C, Lou Y, Xi W, Wang X, Wang Y, Qu Z, Guo C, Chen Y and Zhang Y: Tissue-specific expression of TIPE2 provides insights into its function. Mol Immunol 47: 2435-2442, 2010.

20. Dull T, Zufferey R, Kelly M, Mandel RJ, Nguyen M, Trono D and Naldini L: A third-generation lentivirus vector with a conditional packaging system. J Virol 72: 8463-8471, 1998

21. Wu J, Zhang H, Xu C, Xu H, Zhou X, Xie Y and Tao M: TIPE2 functions as a metastasis suppressor via negatively regulating $\beta$-catenin through activating GSK $3 \beta$ in gastric cancer. Int J Oncol 48: 199-206, 2016

22. Cao X, Zhang L, Shi Y, Sun Y, Dai S, Guo C, Zhu F, Wang Q, Wang J, Wang X, et al: Human tumor necrosis factor (TNF)alpha-induced protein 8-like 2 suppresses hepatocellular carcinoma metastasis through inhibiting Rac1. Mol Cancer 12: 149, 2013.

23. Lauring J,Park BH and Wolff AC: The phosphoinositide-3-kinaseAkt-mTOR pathway as a therapeutic target in breast cancer. J Natl Compr Canc Netw 11: 670-678, 2013.

24. Yang SX, Polley E and Lipkowitz S: New insights on PI3K/AKT pathway alterations and clinical outcomes in breast cancer. Cancer Treat Rev 45: 87-96, 2016.

25. Chen L, Mayer JA, Krisko TI, Speers CW, Wang T, Hilsenbeck SG and Brown PH: Inhibition of the p38 kinase suppresses the proliferation of human ER-negative breast cancer cells. Cancer Res 69: 8853-8861, 2009.

26. Kim MS, Lee EJ, Kim HR and Moon A: p38 kinase is a key signaling molecule for $\mathrm{H}$-Ras-induced cell motility and invasive phenotype in human breast epithelial cells. Cancer Res 63: 5454-5461, 2003.

27. Liu C, Wang S, Zhu S, Wang H, Gu J, Gui Z, Jing J, Hou X and Shao Y: MAP3K1-targeting therapeutic artificial miRNA suppresses the growth and invasion of breast cancer in vivo and in vitro. Springerplus 5: 11, 2016. 